\title{
GROUPS IN WHICH EVERY MAXIMAL PARTIAL ORDER IS EXHAUSTIVE ${ }^{1}$
}

\author{
GARY J. SHERMAN
}

\begin{abstract}
Let $G$ be a group. A partial order $P$ on $G$ is exhaustive if each nongeneralized periodic element of $G$ is either positive or negative with respect to $P$. The class of groups on which every maximal partial order is exhaustive is characterized and shown to be closed under homomorphisms. An example is given to show that the class of groups on which every maximal right partial order is exhaustive is not closed under direct products.
\end{abstract}

The normal subsemigroup generated by an element $x$ of the group $G$ is denoted by $S(x)$. An element $x$ is said to be generalized periodic (g.p.) if $e \in S(x)$ and nongeneralized periodic (n.g.p.) otherwise. A partial order on $G$ is exhaustive if each n.g.p. element is either positive or negative. The group $G$ is said to be an $E^{*}$-group if each partial order on $G$ can be extended to an exhaustive partial order.

If every element of $G$ is n.g.p., then $G$ is an $E^{*}$-group if and only if it is an $O^{*}$-group (i.e. a group in which each partial can be extended to a full order). Ohnishi [5] has shown that a group $G$ is an $O^{*}$-group if, and only if, it satisfies the following two conditions:

(i) Every nontrivial element is g.p.

(ii) For all $x, y$ and $z$ in $G$, if $x \in S(z)$ and $y \in S(z)$ then $S(x) \cap S(y) \neq \varnothing$. Hollister [4] has studied the class of groups satisfying (ii). We characterize the class of $E^{*}$-groups with the following generalization of (ii).

(iii) For each n.g.p. element $z$, if $x, y \in S(z)$, then $S(x) \cap S(y) \neq \varnothing$.

THeOREM 1. A group $G$ is an $E^{*}$-group if, and only if, it satisfies condition (iii).

Presented to the Society, January 17, 1972 under the title On exhaustively ordered groups, received by the editors November 7, 1972.

AMS (MOS) subject classifications (1970). Primary 06A55; Secondary $20 \mathrm{E} 15$.

Key words and phrases. Exhaustively ordered group, partially ordered group, generalized group periodicity, nilpotent group.

${ }^{1}$ This paper is based in part on a portion of the author's doctoral dissertation (Indiana University) which was written under the direction of Professor Maria J. Wonenburger. The preparation of the paper was supported by a Rose-Hulman Institute of Technology Summer Fellowship. 
Proof. Assume that $G$ satisfies condition (iii) and let $P$ be a maximal partial order on $G$. If $z$ is n.g.p. and $z \notin P \cup P^{-1}$ then $S(z) \cap P \neq \varnothing$. For if $S(z) \cap P=\varnothing$, then $P \cdot S\left(z^{-1}\right)$ is a partial order on $G$. Due to the maximality of $P$, we obtain the contradiction $z^{-1} \in P$. Similarly, $S(z) \cap P^{-1} \neq \varnothing$. Then $S(x) \subseteq S(z) \cap P$ and $S(y) \subseteq S(z) \cap P^{-1}$ for some $x, y \in G$ and so $\varnothing \neq S(x) \cap S(y) \subseteq P \cap P^{-1}$. Therefore $P \cap P^{-1} \neq e$, a contradiction.

Conversely, let $G$ be an $E^{*}$-group and suppose $x, y \in S(z)$ where $z$ is n.g.p. If $S(x) \cap S(y)=\varnothing$, then $S^{\prime}(x) \cdot S^{\prime}\left(y^{-1}\right)$ is a partial order that can be extended to an exhaustive partial order $P$. Then $x, y^{-1} \in P$ and so $z, z^{-1} \in P$, a contradiction.

It is a corollary of the preceding theorem that an abelian group is an $E^{*}$-group. Thus an abelian group is an $O^{*}$-group if, and only if, it is torsion-free (Simbireva [6]). Since there exist torsion-free abelian groups with nontrivial homomorphic images which are not torsion-free it follows that a homomorphic image of an $O^{*}$-group is not necessarily an $O^{*}$ group.

THEOREM 2. A homomorphic image of an $E^{*}$-group is an $E^{*}$-group.

Proof. Let $N$ be a normal subgroup of the $E^{*}$-group $G$. Let $x N, y N$ and $z N$ be n.g.p. elements of $G / N$ and assume $x N, y N \in S(z N)$. Since $S(z N)=S(z) \cdot N$ there exist $n, m \in N$ such that $x n, y m \in S(z)$. It is easy to verify that $x n, y m$ and $z$ are n.g.p. elements of $G$. Theorem 1 implies that $S(x n) \cap S(y m) \neq \varnothing$. Hence there exists $w_{1}, \cdots, w_{k}, v_{1}, \cdots, v_{q} \in G$ for which $\prod_{i=1}^{k}(x n)^{w_{i}}=\prod_{j=1}^{q}(y m)^{v_{j}}$. It follows that $\left(\prod_{i=1}^{k} x^{w_{i}}\right) n_{1}=$ $\left(\prod_{j=1}^{q} y^{v_{j}}\right) m_{1}$ where $n_{1}, m_{1} \in N$. Thus $\left(\prod_{i=1}^{k} x^{w_{i}}\right) N=\left(\prod_{j=1}^{q} y^{v_{j}}\right) N$ and since both $x N$ and $y N$ are n.g.p. we have that $S(x N) \cap S(y N) \neq \varnothing$.

Corollary 3. A homomorphic image of an $O^{*}$-group is an $E^{*}$-group.

THEOREM 4. Let $N$ denote the set of generalized periodic elements of the group $G$. If $N$ forms a subgroup, then $G$ is an $E^{*}$-group if, and only if, $G / N$ is an $O^{*}$-group.

Proof. Let $G$ be an $E^{*}$-group. Theorem 2 implies that $G / N$ is an $E^{*-}$ group. Since every element of $G / N$ is n.g.p., it must be an $O^{*}$-group.

If $P$ is a partial order on $G$, then $N P / N$ is a partial order on $G / N$. By assumption, there exists a full order $(N P / N)^{*}$ on $G / N$ which is an extension of $N P / N$. The lexicographic extension of $e$ by $(N P / N)^{*}$ is an exhaustive partial order on $G$ extending $P$.

COROLlary 5. A locally nilpotent group is an $E^{*}$-group.

Proof. In this case $N$ is a subgroup and $G / N$ is torsion free and locally nilpotent and hence an $O^{*}$-group. 
Statements analogous to Theorem 4 are valid for three classes of groups containing the $E^{*}$-groups. A group which admits an exhaustive partial order is called an $E$-group. A torsion-free $E$-group is an $O$-group (i.e., a group which admits a full order).

THEOREM 6. Let $N$ denote the set of generalized periodic elements of the group $G$. If $N$ forms a subgroup, then $G$ is an E-group if, and only if, $G / N$ is an $O$-group.

A right partial order on $G$ is exhaustive if every element of infinite order in $G$ is positive or negative. A group which admits an exhaustive right partial order is an $E_{r}$-group while a group in which every right partial order can be extended to an exhaustive right partial order is an $E_{r}^{*}$-group. The definitions of $O_{r}$-groups and $O_{r}^{*}$-groups are obvious.

THEOREM 7. Let $F$ denote the set of periodic elements of the group $G$. If $F$ is a subgroup, then $G$ is an $E_{r}^{*}$-group $\left(E_{r}\right.$-group) if, and only if, $G / F$ is an $O_{r}^{*}$-group $\left(O_{r}\right.$-group $)$.

The proofs of Theorems 6 and 7 essentially amount to observing that $N P / N(F P / F)$ is a full order (right full order) if $P$ is an exhaustive partial order (exhaustive right partial order), and conversely.

Fuchs [3] has shown that the direct product of two $O^{*}$-groups is again an $O^{*}$-group. Hollister [4] extended this result to the class of groups satisfying condition (ii). It is not known whether the direct product of two $E^{*}$-groups is an $E^{*}$-group. We provide an example to show that the direct product of two $E_{r}^{*}$-groups is not, in general, an $E_{r}^{*}$-group.

The free product of the cyclic group of order two with itself is denoted by $Z_{2} * Z_{2}$. This group has the presentation: $\left\langle a, b: a^{2}=e\right.$ and $\left.b^{2}=e\right\rangle$. The elements of infinite order together with the identity, denoted by $I_{e}$, form an infinite cyclic group generated by $a b$.

Fact 1. $Z_{2} * Z_{2}$ is an $E_{r}^{*}$-group.

Proof. Let $P$ be a maximal right partial order on $Z_{2} * Z_{2}$. Since $P \subseteq I_{e}, P$ is a maximal partial order on the cyclic group $I_{e}$. Thus, $P$ is a full order on $I_{e}$. It follows that $P$ is an exhaustive right partial order on $Z_{2} * Z_{2}$.

There are two full orders on $I_{e}$. Let $R$ be the one for which $a b$ is positive.

Fact 2. $\left(Z_{2} * Z_{2}\right) \times\left(Z_{2} * Z_{2}\right)$ is not an $E_{r}^{*}$-group.

PROOF. The lexicographic product $L$ of $R$ with itself is a right partial order on $\left(Z_{2} * Z_{2}\right) \times\left(Z_{2} * Z_{2}\right)$. Suppose $Q$ is an exhaustive right partial order which is an extension of $L$. Since $(a, b a)$ has infinite order we must have $(a, b a) \in Q$ or $(a, a b) \in Q$. From the definition of $L$ it follows that $(a b, a) \in L$. If $(a, b a) \in Q$, then $(a, b a)(a b, a)=(b, b) \in Q$, a contradiction 
since $(b, b)$ is periodic. Similarly, if $(a, a b) \in Q$ we arrive at a contradiction. Thus $L$ can not be extended to an exhaustive right partial order.

It is easily verified that $Z_{2} * Z_{2}$ is a solvable group. A solvable series for $Z_{2} * Z_{2}$ is: $Z_{2} * Z_{2} \supsetneqq I_{e} \supsetneqq e$. Since the direct product of two solvable groups is a solvable group, it follows from the previous fact that a solvable group is not necessarily and $E_{r}^{*}$-group. It is not known whether a nilpotent group must be an $E_{r}^{*}$-group. To solve this problem, it suffices to determine whether a torsion-free nilpotent group must be an $O_{r}^{*}$-group. Ault [1] has shown that a torsion-free nilpotent group of class 2 is an $O_{r}^{*}$-group. The problem is open for nilpotent groups of higher class.

\section{BIBLIOGRAPHY}

1. J. C. Ault, Extensions of partial right orders on nilpotent groups, J. London Math. Soc. (2) 2 (1970), 749-752. MR 42 \#5874.

2. L. Fuchs, Partially ordered algebraic systems, Pergamon Press, New York; Addison-Wesley, Reading, Mass., 1963. MR 30 \#2090.

3. - On orderable groups, Proc. Internat. Conf. Theory of Groups (Austral. Nat. Univ. Canberra, 1965), New York, 1966, pp. 89-98.

4. H. A. Hollister, On a condition of Ohnishi, Proc. Amer. Math. Soc. 19 (1968), 1337-1340. MR 38 \#1045.

5. M. Ohnishi, On linearization of ordered groups, Osaka Math. J. 2 (1950), 161-164. MR 13, 436.

6. H. Simbireva, On the theory of partially ordered groups, Mat. Sb. 20 (62) (1947), 145-178. (Russian) MR 8, 563.

Department of Mathematics, Rose-Hulman Institute of Technology, Terre HAUTE, INDIANA 47803 